\title{
Tensor Components and indices of faintness for magneto-electric ferroic species
}

\author{
Prof. S. Uma Devi \& *G. Sireesha \\ Department of Engineering Mathematics, College of Engineering,Andhra University, Visakhapatnam - 530003.
}

\begin{abstract}
The concept of ferroic species, prototypic and ferroic point groups, and evaluation of faintness indices for all the zero wave number vibrational modes whose oversoftening causes ferroelectricity (or) ferroelasticity are given by Aizu ${ }^{[4]}$. Aizu has dealt with non-magnetic properties only. This paper determines the species and evaluates the indices of faintness for magneto-electric polarizability by considering grey group as prototypic point group. The basic faintness index of a magneto-electric polarizability is the smallest of the faintness indices with respect to the individual components of the magneto-electric polarizability tensors. The faintness indices range from 1 to $6^{[5]}$, whether it is $=1($ or $)>1$ defines whether the magneto-electric polarizability is normal (or) faint.
\end{abstract}

Key Words: Axial vector, grey group, magneto-electric polarizability, polar vector

\section{Introduction:}

The difference between the ordinary point groups and the grey point groups is that in the former the antisymmetry operation $R_{2}$ is not present at all, whereas in the latter it is an operation of the group and has the effect of doubling the order of the point group. Since $\mathrm{R}_{2}$ commutes with all the elements of the point group $\mathrm{G}^{[2]}$, the grey groups are therefore direct product groups of $G$ and $1^{1}\left(1^{1}\right.$ is a group consisting of identity and time inversion operation $\mathrm{R}_{2}$ ) which is designated as $\mathrm{G} 1^{1}$. Magneto-electric polarizability is the production of magnetic moment $\mathrm{I}$ on the application of an electric field $\mathrm{E}$ in a direction normal to $\mathrm{it}^{[1]}$. The relation between $\mathrm{E}$ and $I$ is given by $I_{i}=\lambda_{i j} E_{j}$. where $E$ is a polar vector and its components are $(x, y, z)$. I is an axial vector and its components are $\left(\mathrm{x}^{1}, \mathrm{y}^{1}, \mathrm{z}^{1}\right) . \quad \lambda_{\mathrm{ij}}$ is the magneto-electric polarizability tensor (or) a second rank tensor which transforms according to the representation formed by the product of the representations of polar and axial vectors $^{[3]}$ which can be expressed as a matrix in the form of either $\left[\begin{array}{lll}x x^{1} & x y^{1} & x z^{1} \\ y x^{1} & y y^{1} & y z^{1} \\ z x^{1} & z y^{1} & z z^{1}\end{array}\right]$ (or) $\left[\begin{array}{lll}\lambda_{11} & \lambda_{12} & \lambda_{13} \\ \lambda_{21} & \lambda_{22} & \lambda_{23} \\ \lambda_{31} & \lambda_{32} & \lambda_{33}\end{array}\right]$.

The character for magneto-electric polarizability is given by Bhagavantham and Pantulu (1964) is $\chi^{1}(\mathrm{R})=$ $(1 \pm 2 \cos \phi)(2 \cos \phi \pm 1)$. In this equation + ve sign (or) -ve sign is to be taken accordingly as symmetry operation $\mathrm{R}$ is a pure rotation (or) a rotation-reflection. When the prototypic point group is a grey group, the ferroic species are associated to the corresponding irreducible representations of the grey groups and their corresponding components with faintness indices are tabulated. As an example the grey group $4 \mathrm{~mm} 1{ }^{1}$ is illustrated.

\section{Example:}

Irreducible representations for the grey group $4 \mathrm{~mm} 1^{1}$ are $\mathrm{A}_{1}{ }^{1}, \mathrm{~A}_{2}{ }^{1}, \mathrm{~B}_{1}{ }^{1}, \mathrm{~B}_{2}{ }^{1}$ and $\mathrm{E}$. Consider the irreducible representation $\mathrm{A}_{2}{ }^{1}$, its corresponding ferroic species is $4 \mathrm{~mm} 1^{1} \mathrm{~F} 4 \mathrm{~m}^{1} \mathrm{~m}^{1}$. The components which are invariant for this species are $\left(x x^{1}+y y^{1} / 2, z z^{1}\right)$. For this species the value of $\mathrm{n}_{\mathrm{i}}$ against that representation to which the species is associated is 2 . These 2 components appear directly. Hence the index of faintness for this species is normal i.e. 1. For the 2-dimensional representation E, its corresponding ferroic species are $4 \mathrm{~mm} 1^{1} \mathrm{~F} 2^{1}, 4 \mathrm{~mm} 1^{1} \mathrm{~F} 1$. The 4 components for the species $4 \mathrm{~mm} 1^{1} \mathrm{~F}^{1}$ appear directly. Hence the index of faintness for this species is 1 . For the species $4 \mathrm{~mm} 1{ }^{1} \mathrm{~F} 1$, it has 9 components. Out of 9 components 4 components appear directly and their index of faintness is 1 . The remaining 5 components does not appear directly. They appear for the symmetrized cube of the property. Hence their index of faintness for these 5 components is 3 and these are listed in table 1 . 
Table 1:

\begin{tabular}{|c|c|l|c|}
\hline $\begin{array}{c}\text { Grey } \\
\text { Group }\end{array}$ & $\begin{array}{c}\text { Irreducible } \\
\text { representations }\end{array}$ & $\begin{array}{c}\text { Ferroic } \\
\text { species }\end{array}$ & $\begin{array}{c}\text { Tensor components and indices of faint- } \\
\text { ness for magneto-electric polarizability }\end{array}$ \\
\hline 4mm1 & $\mathrm{A}_{1}{ }^{1}$ & $4 \mathrm{~mm} 1^{1} \mathrm{~F} 4 \mathrm{~mm}$ & $\left(x y^{1}\right) \mathbf{1}$ \\
\hline & $\mathrm{A}_{2}{ }^{1}$ & $4 \mathrm{~mm} 1^{1} \mathrm{~F} 4 \mathrm{~m}^{1} \mathrm{~m}^{1}$ & $\left(\frac{x x^{1}+y y^{1}}{2}, z z^{1}\right) \mathbf{1}$ \\
\hline & $\mathrm{B}_{1}{ }^{1}(\mathrm{i}=1,2)$ & $4 \mathrm{~mm} 1^{1} \mathrm{~F} 4^{1} \mathrm{~mm}^{1}$ & $\left(x y^{1}, y x^{1}\right) \mathbf{1}$ \\
\hline & $\mathrm{E}$ & $4 \mathrm{~mm} 1^{1} \mathrm{~F} 2^{1}$ & $\left(x z^{1}, y z^{1}, z x^{1}, z y^{1}\right) \mathbf{1}$ \\
\hline & $4 \mathrm{~mm} 1^{1} \mathrm{~F} 1$ & $\begin{array}{l}\left(x z^{1}, y z^{1}, z x^{1}, z y^{1}\right) \mathbf{1} \\
\left(x x^{1}, x y^{1}, y x^{1}, y y^{1}, z z^{1}\right) \mathbf{3}\end{array}$ \\
\hline
\end{tabular}

Similarly the components and the indices of faintness for magneto-electric polarizability are evaluated for all 32 grey groups and the results are tabulated in table 2.

Table 2:

\begin{tabular}{|c|c|c|c|}
\hline $\begin{array}{l}\text { Grey } \\
\text { Group }\end{array}$ & \begin{tabular}{|c|}
$\begin{array}{c}\text { Irreducible } \\
\text { representations }\end{array}$ \\
\end{tabular} & $\begin{array}{l}\text { Ferroic } \\
\text { species }\end{array}$ & $\begin{array}{l}\text { Tensor components and indices of faint- } \\
\text { ness for magneto-electric polarizability }\end{array}$ \\
\hline \multirow[t]{2}{*}{$\overline{1} 1^{1}$} & $\mathrm{~A}^{1}$ & $\overline{11} 1^{1} \mathrm{~F} 1$ & 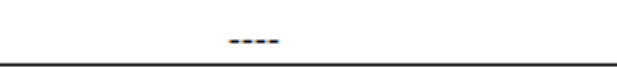 \\
\hline & $\mathrm{B}^{1}$ & $\overline{1} 1^{1} \mathrm{Fi}^{-1}$ & $\left(x x^{1}, x y^{1}, x z^{1}, y x^{1}, y y^{1}, y z^{1}, z x^{1}, z y^{1}, z z^{1}\right) \mathbf{1}$ \\
\hline \multirow[t]{2}{*}{$21^{1}$} & $\mathrm{~A}^{1}$ & $21^{1} \mathrm{~F} 2$ & $\left(x x^{1}, x y^{1}, y x^{1}, y y^{1}, z z^{1}\right) \mathbf{1}$ \\
\hline & $\mathrm{B}^{1}$ & $21^{1} \mathrm{~F} 2^{1}$ & $\left(x z^{1}, y z^{1}, z x^{1}, z y^{1}\right) \mathbf{1}$ \\
\hline \multirow[t]{2}{*}{$\mathrm{ml}^{1}$} & $\mathrm{~A}_{1}{ }^{1}$ & $\mathrm{~m}^{1}{ }^{1} \mathrm{Fm}$ & $\left(x z^{1}, y z^{1}, z x^{1}, z y^{1}\right) \mathbf{1}$ \\
\hline & $\mathrm{A}_{1}^{11}$ & $\mathrm{~m}^{1} \mathrm{Fm}^{1}$ & $\left(x x^{1}, x y^{1}, y x^{1}, y y^{1}, z z^{1}\right) \mathbf{1}$ \\
\hline \multirow[t]{4}{*}{$2 / \mathrm{ml}^{1}$} & $\mathrm{Ag}_{\mathrm{g}}{ }^{1}$ & $2 / \mathrm{m}^{1} \mathrm{~F} 2 / \mathrm{m}$ & --- \\
\hline & $\mathrm{Bg}_{\mathrm{g}}{ }^{\mathrm{B}}$ & $2 / \mathrm{m}^{1} \mathrm{~F} 2^{1} / \mathrm{m}^{1}$ & --- \\
\hline & $\mathrm{A}_{\mathrm{u}}{ }^{1}$ & $2 / \mathrm{m}^{1}{ }^{1} \mathrm{~F} 2 / \mathrm{m}^{1}$ & $\left(x x^{1}, x y^{1}, y x^{1}, y y^{1}, z z^{1}\right) \mathbf{1}$ \\
\hline & $\mathrm{B}_{\mathrm{u}}{ }^{1}$ & $2 / \mathrm{m}^{1}{ }^{1} \mathrm{~F} 2^{1} / \mathrm{m}$ & $\left(x z^{1}, y z^{1}, z x^{1}, z y^{1}\right) \mathbf{1}$ \\
\hline \multirow[t]{2}{*}{$2221^{1}$} & $\mathrm{~A}^{1}$ & $2221^{1} \mathrm{~F} 222$ & $\left(x x^{1}, y y^{1}, z z^{1}\right) \mathbf{1}$ \\
\hline & $\mathrm{B}_{\mathrm{i}}^{1}(\mathrm{i}=1,2,3)$ & $2221^{1} \mathrm{~F} 2^{1} 2^{1} 2$ & $\left(x y^{1}, y x^{1}\right) \mathbf{1}$ \\
\hline \multirow[t]{3}{*}{$\mathrm{mm} 21^{1}$} & $\mathrm{~A}_{1}{ }^{1}$ & $\mathrm{~mm} 21^{1} \mathrm{Fmm} 2$ & $\left(x y^{1}, y x^{1}\right) \mathbf{1}$ \\
\hline & $\mathrm{A}_{2}{ }^{1}$ & $\mathrm{~mm} 21^{1} \mathrm{Fm} \mathrm{m}^{1} \mathrm{~m}^{1} 2$ & $\left(x x^{1}, y y^{1}, z z^{1}\right) \mathbf{1}$ \\
\hline & $B_{i}^{1}(i=1,2)$ & $\mathrm{mm} 21^{1} \mathrm{Fmm}^{1} 2^{1}$ & $\left(x z^{1}, z x^{1}\right) \mathbf{1}$ \\
\hline \multirow[t]{4}{*}{$\mathrm{mmml}^{1}$} & $\mathrm{Ag}_{\mathrm{g}}{ }^{1}$ & $\mathrm{mmm}^{1}{ }^{1} \mathrm{Fmmm}$ & $\ldots$ \\
\hline & $B_{i g}{ }^{1}(i=1,2,3)$ & $\mathrm{mmm}^{1} 1^{1} \mathrm{Fm}^{1} \mathrm{~m}^{1} \mathrm{~m}$ & $-\cdots$ \\
\hline & $\mathrm{A}_{\mathrm{u}}{ }^{1}$ & $\mathrm{mmm} 1^{1} \mathrm{Fm}^{1} \mathrm{~m}^{1} \mathrm{~m}^{1}$ & $\left(x x^{1}, y y^{1}, z z^{1}\right) \mathbf{1}$ \\
\hline & $\mathrm{B}_{\mathrm{iu}}{ }^{1}(\mathrm{i}=1,2,3)$ & $\mathrm{mmm}^{1} \mathrm{Fmm}^{1} \mathrm{~m}$ & $\left(x y^{1}, y x^{1}\right) \mathbf{1}$ \\
\hline \multirow[t]{2}{*}{$41^{1}$} & $\mathrm{~A}^{1}$ & $41^{1} \mathrm{~F} 4$ & $\left(x x^{1}, y y^{1}, z z^{1}\right) \mathbf{1}$ \\
\hline & $\mathrm{B}^{1}$ & $41^{1} \mathrm{~F} 4^{1}$ & $\left(x y^{1}, y x^{1}\right) \mathbf{1}$ \\
\hline
\end{tabular}


Tensor Components and indices of faintness for magneto-electric ferroic species

\begin{tabular}{|c|c|c|c|}
\hline & $\mathrm{E}^{1}$ & $41^{t} \mathrm{~F} 2^{!}$ & $\left(x z^{1}, y z^{1}, z x^{1}, z y^{1}\right) 1$ \\
\hline \multirow[t]{3}{*}{$\overline{4} 1^{1}$} & $A^{1}$ & $\overline{4} 1 ! 5 \overline{4}$ & $\left(x y^{1}, u x^{1}\right)_{1}$ \\
\hline & $\mathrm{B}^{\mathrm{t}}$ & $\overline{4} 1^{1} \mathrm{~F}^{-1}$ & $\left(x x^{1}, y y^{1}, z z^{1}\right)_{1}$ \\
\hline & $\mathrm{E}^{1}$ & $\overline{4} 1^{1} \mathrm{~F} 2^{1}$ & $\left(x z^{1}, y z^{1}, z x^{1}, z y^{1}\right) 1$ \\
\hline \multirow[t]{6}{*}{$4 / \mathrm{ml}^{1}$} & $\mathrm{Ag}^{1}$ & $4 / \mathrm{ml}^{\mathrm{i}} \mathrm{F} 4 / \mathrm{m}$ & $\ldots$ \\
\hline & $\mathrm{Bg}^{1}$ & $4 / \mathrm{ml}^{1} \mathrm{~F} 4^{1} / \mathrm{m}$ & $\ldots$ \\
\hline & $\mathrm{Eg}^{1}$ & $4 / \mathrm{ml}^{1} \mathrm{~F} 2^{1} / \mathrm{m}^{1}$ & $\ldots$ \\
\hline & $\mathrm{Au}^{2}$ & $4 / \mathrm{ml}^{1} \mathrm{~F} 4 / \mathrm{m}^{\mathrm{l}}$ & $\left(x x^{1}, y y^{1}, z z^{1}\right) 1$ \\
\hline & $\mathrm{Bu}^{1}$ & $4 / \mathrm{ml}^{1} \mathrm{~F} 4^{1} / \mathrm{m}^{1}$ & $\left(x y^{1}, u x^{2}\right) 1$ \\
\hline & $\mathrm{Eu}^{\mathrm{t}}$ & $4 / \mathrm{ml}^{1} \mathrm{~F} 2^{1} / \mathrm{m}$ & $\left(x z^{1}, y z^{1}, z x^{1}, z y^{1}\right){ }_{1}$ \\
\hline \multirow[t]{5}{*}{$4221^{1}$} & $A_{1}^{1}$ & $4221^{1} \mathrm{~F} 422$ & $\left(\frac{x x^{1}+y y^{2}}{2}, z z^{1}\right) 1$ \\
\hline & $\mathrm{A}_{2}{ }^{1}$ & $4221^{t} \mathrm{~F} 42^{\mathrm{l}} 2^{\mathrm{l}}$ & $\left(x y^{1}\right)$ where $\left(\lambda_{21}=-\lambda_{12}\right) 1$ \\
\hline & $\mathrm{B}_{\mathrm{i}}^{\mathrm{t}}(\mathrm{i}=1,2)$ & $4221^{1} \mathrm{~F} 4^{!} 2^{\mathrm{i}} 2$ & $\left(x y^{1}, w x^{1}\right) 1$ \\
\hline & $\mathrm{E}_{1}^{\mathrm{t}}$ & $4221^{\mathrm{F}} \mathrm{F} 2^{\mathrm{t}}$ & $\left(x z^{1}, y z^{1}, z x^{1}, z y^{1}\right)_{1}$ \\
\hline & & $4221^{t} \mathrm{Fl}$ & $\begin{array}{l}\left(x z^{1}, y z^{1}, z x^{1}, z y^{1}\right)_{1} \\
\left(x x^{1}, x y^{1}, y x^{1}, y y^{1}, z z^{1}\right)_{3}\end{array}$ \\
\hline \multirow[t]{5}{*}{$4 \mathrm{mml}^{1}$} & $\mathrm{~A}_{\mathrm{t}}{ }^{\mathrm{I}}$ & $4 \mathrm{mml}^{1} \mathrm{~F} 4 \mathrm{~mm}$ & $\left(x y^{2}\right) 1$ \\
\hline & $\mathrm{A}_{2}{ }^{\mathrm{I}}$ & $4 \mathrm{mml}^{1} \mathrm{~F} 4 \mathrm{~m}^{1} \mathrm{~m}^{\mathrm{t}}$ & $\left(\frac{x x^{1}+y^{1}}{2}, z z^{1}\right) 1$ \\
\hline & $\mathrm{B}_{\mathrm{i}}^{\mathrm{l}}(\mathrm{i}=1,2)$ & $4 \mathrm{mml}^{1} \mathrm{~F} 4^{1} \mathrm{~mm}^{1}$ & $\left(x y^{1}, u x^{1}\right) 1$ \\
\hline & $\mathrm{E}$ & $4 \mathrm{mml}^{1} \mathrm{~F} 2^{\mathrm{I}}$ & $\left(x z^{1}, y z^{1}, z x^{1}, z y^{1}\right) 1$ \\
\hline & & $4 \mathrm{mml}^{1} \mathrm{Fl}$ & $\begin{array}{l}\left(x z^{1}, y z^{1}, z x^{1}, z y^{1}\right)_{1} \\
\left(x x^{1}, x y^{1}, y x^{2}, w y^{1}, z z^{1}\right)_{3}\end{array}$ \\
\hline \multirow[t]{3}{*}{$\overline{4} 2 \mathrm{~m} 1$} & $A_{1}{ }^{2}$ & $\overline{4} 2 \mathrm{ml}^{1} \mathrm{~F} \overline{4} 2 \mathrm{~m}$ & $\left(x x^{1}\right)$ where $\left(\lambda_{2}=-\lambda_{11}\right) 1$ \\
\hline & $\mathrm{A}_{2}{ }^{\mathrm{I}}$ & $\overline{4} 2 \mathrm{ml}^{1} \mathrm{~F} \overline{4} 2^{1} \mathrm{~m}^{\mathrm{t}}$ & $\left(\frac{x y^{1}+y x^{1}}{2}\right) \quad 1$ \\
\hline & $\mathrm{B}_{1}^{\mathrm{t}}$ & $\overline{4} 2 \mathrm{ml}^{\mathrm{l}} \mathrm{F} \overline{4}^{1} 2 \mathrm{~m}^{1}$ & $\left(\frac{x x^{1}+y y^{1}}{2}, z z^{1}\right)^{1}$ \\
\hline
\end{tabular}




\begin{tabular}{|c|c|c|c|}
\hline \multicolumn{2}{|r|}{$\mathrm{B}_{2}^{2}$} & \multirow{2}{*}{$\frac{\overline{4} 2 \mathrm{mll}^{2} \mathrm{~F} \overline{4}^{2} 2^{2} \mathrm{~m}}{\overline{4} 2 \mathrm{ml}^{2} \mathrm{~F} 2^{2}}$} & \multirow{2}{*}{$\frac{\left(x y^{2}\right) \text { where }\left(\lambda_{21}=-\lambda_{12}\right)}{\mid\left(x z^{2}, v z^{2}, z x^{2}, z v^{2}\right)} 1$} \\
\hline & $E^{2}$ & & \\
\hline & & $\overline{4} 2 \mathrm{ml}^{2} \mathrm{~F} 1$ & $\begin{array}{l}\left(x z^{2}, y z^{2}, 2 x^{2}, x y^{2}\right) 1^{1} \\
\left(x x^{2}, x y^{2}, v x^{2}, w w^{2}, z z^{2}\right)^{3}\end{array}$ \\
\hline \multirow[t]{8}{*}{ 4/mmml } & $A_{1 z^{2}}$ & $4 / \mathrm{mmml}^{2} \mathrm{~F} 4 / \mathrm{mmm}$ & $\ldots$ \\
\hline & $\mathbf{A}_{2 z^{2}}$ & $4 / \mathrm{mmm}^{2} \mathrm{I}^{2} \mathrm{~h} / \mathrm{m}^{2} \mathrm{~m}^{2} \mathrm{~m}$ & $\ldots$ \\
\hline & $B_{s}{ }^{2}(i=1,2)$ & $4 / \mathrm{mmm} 1^{2} \mathrm{~F}^{2} / \mathrm{m}^{2} \mathrm{~mm}$ & $\ldots$ \\
\hline & $\mathrm{E}_{z}{ }^{2}$ & $4 / \mathrm{mmm} 1^{2} \mathrm{~F} 2^{1} / \mathrm{m}^{2}$ & $\ldots$ \\
\hline & $A_{i=u^{2}}$ & $4 / m m m 1^{2} F 4 / m^{2} m^{2} m^{2}$ & $\left(\frac{x x^{1}+y y^{2}}{2}, z z^{2}\right) 1$ \\
\hline & $A_{2 u^{2}}{ }^{2}$ & $4 / \mathrm{mmml}^{2} \mathrm{~F} 4 / \mathrm{m}^{2} \mathrm{~mm}$ & $\left(x y^{2}\right)$ where $\left(\lambda_{n 1}=-\lambda_{12}\right) 1$ \\
\hline & $B_{s=}^{2}(i=1,2)$ & $4 / m m m 1^{2} F 4^{2} / m^{2} m^{2} m$ & $\left(x y^{2}, y x^{2}\right) 1$ \\
\hline & $E_{s}{ }^{2}$ & $4 / m m m 1^{2} F 2^{2} / m$ & $\left(x z^{2}, y z^{2}, z x^{2}, z y^{2}\right) \quad 1$ \\
\hline \multirow[t]{2}{*}{$31^{1}$} & $A^{2}$ & $31^{2} \mathrm{~F} 3$ & $\left(\frac{x x^{2}+y y^{2}}{2}, x v^{2}, z z^{2}\right) 1$ \\
\hline & $\mathrm{E}^{2}$ & $31^{2} \mathrm{~F} 1$ & $\begin{array}{l}\left(x z^{2}, v x^{2}, y y^{2}, y z^{2}, x x^{2}, z y^{2}\right) 1 \\
\left(x x^{2}, x y^{2}, z z^{1}\right) 3\end{array}$ \\
\hline \multirow[t]{4}{*}{$31^{1}$} & $A_{z^{2}}{ }^{2}$ & $31^{2} \mathrm{F3}$ & $\ldots$ \\
\hline & $\mathrm{E}_{z}{ }^{2}$ & $31^{2} \mathrm{Fi}$ & $\ldots$ \\
\hline & $A_{x}{ }^{2}$ & $3 \mathrm{I}^{2} \mathrm{~F} \overline{3}^{2}$ & $\left(\frac{x x^{2}+y y^{2}}{2}, x y^{2}, z z^{2}\right) 1$ \\
\hline & $E_{s}{ }^{2}$ & $\overline{3} 1^{2} \mathrm{Fi}^{2}$ & $\begin{array}{l}\left(x z^{2}, y x^{2}, y y^{2}, y z^{2}, z x^{2}, z y^{2}\right) 1 \\
\left(x x^{2}, x y^{2}, z z^{2}\right) 3\end{array}$ \\
\hline \multirow[t]{3}{*}{$321^{1}$} & $\mathbf{A}_{2}{ }^{2}$ & $321^{1} \mathrm{~F} 32$ & $\left(\frac{x x^{2}+y y^{2}}{2}, z z^{2}\right) 1$ \\
\hline & $A_{2}{ }^{2}$ & $321^{1} \mathrm{~F} 32^{2}$ & $\left(x v^{2}\right)$ where $\left(\lambda_{21}=-\lambda_{2 z}\right) 1$ \\
\hline & $\mathrm{E}^{2}$ & $321^{2} \mathrm{~F} 1$ & $\begin{array}{l}\left(x x^{2}, x z^{2}, y x^{2}, y z^{2}, z x^{2}, x y^{2}\right) 1^{1} \\
\left.\left(v y^{2}, x y^{2}, z z^{2}\right)\right]^{3}\end{array}$ \\
\hline $3 \mathrm{ml}^{1}$ & $\mathbf{A}_{2}{ }^{2}$ & $3 m 1^{2} F 3 m$ & $\left(x y^{2}\right)$ where $\left(\lambda_{21}=-\lambda_{12}\right) 1$ \\
\hline
\end{tabular}




\begin{tabular}{|c|c|c|c|}
\hline \multicolumn{2}{|r|}{$A_{2}=$} & $3 m I^{2} F 3 m^{2}$ & \multirow{2}{*}{$\begin{array}{l}\left(\frac{x x^{2}+y y^{2}}{2}, z z^{2}\right) \quad 1 \\
\left(x z^{2}, y x^{2}, y y^{2}, y z^{2}, z x^{2}, z y^{2}\right) 1 \\
\left(x x^{2}, x y^{2}, z z^{2}\right) \quad 3\end{array}$} \\
\hline & $\mathbf{E}^{2}$ & $3 m 1^{2} F 1$ & \\
\hline \multirow[t]{5}{*}{$\operatorname{3enT}$} & $A_{1 z^{2}}$ & $3 \mathrm{~min}^{1} \mathrm{~F} 3 \mathrm{~m}$ & $\ldots$ \\
\hline & $A_{2 z^{2}}{ }^{2}$ & $3 \mathrm{mn}^{1} \mathrm{~F} 3 \mathrm{~m}^{2}$ & $\ldots$ \\
\hline & $A_{1=2}=$ & $\overline{3} \mathrm{mll}^{1} \mathrm{~F}^{3} \mathrm{~m}^{2}$ & $\left(\frac{x x^{2}+y y^{2}}{2}, z z^{2}\right) \quad 1$ \\
\hline & $A_{2=x^{2}}$ & $\overline{3} m 1^{1} \mathrm{~F}^{2} \mathrm{~m}$ & $\left(x y^{2}\right)$ where $\left(\lambda_{x 1}=-\lambda_{2 x}\right)$ I \\
\hline & $E_{-2}{ }^{2}$ & $\overline{3} m 1^{2} \mathrm{Fi}^{2}$ & $\begin{array}{l}\left(x x^{2}, x z^{2}, w x^{2}, w z^{2}, 2 x^{2}, 2 y^{2}\right) 1 \\
\left.\left(x y^{2}, 3 y^{2}, 2 z^{2}\right)\right]^{2}\end{array}$ \\
\hline \multirow[t]{4}{*}{$61^{x}$} & $A^{2}$ & $61^{2} \mathrm{~F} 6$ & $\left(\frac{x x^{2}+y y^{2}}{2}, x y^{2}, z z^{2}\right) 1$ \\
\hline & $\mathbf{B}^{2}$ & $61^{2} \mathrm{FG}^{2}$ & $\ldots$ \\
\hline & $E_{2}{ }^{2}$ & $61^{2} F 2^{2}$ & $\left(x z^{2}, y z^{2}, z x^{2}, z y^{2}\right) 1$ \\
\hline & $\mathbf{E}_{2}{ }^{2}$ & $61^{2} F 2$ & $\begin{array}{l}\left(3 x^{2}, x y^{2}\right) 1 \\
\left(x x^{2}, x y^{2}, z z^{2}\right) \quad 3\end{array}$ \\
\hline \multirow[t]{4}{*}{$61^{2}$} & $\mathbf{A}_{2}{ }^{2}$ & $61^{1} \mathrm{~F} 6$ & $\ldots$ \\
\hline & $E_{2}{ }^{2}$ & $61^{1} \mathrm{Fm}$ & $\left(x z^{2}, y z^{2}, z x^{2}, z y^{2}\right) 1$ \\
\hline & $A_{2}=2$ & $\overline{6} \mathbb{1}^{2} \overline{6}^{2}$ & $\left(\frac{x x^{2}+x y^{2}}{2}, x y^{2}, x z^{2}\right) 1$ \\
\hline & $E_{2}{ }^{2}=$ & BII $\mathrm{Fm}^{2}$ & $\begin{array}{l}\left(x x^{2}, 3 v x^{2}\right) \quad 1 \\
\left(x y^{2}, 3 y^{2}, z z^{2}\right) \quad 3\end{array}$ \\
\hline \multirow[t]{6}{*}{$6 / \mathrm{mil}^{1}$} & $\mathrm{Az}_{z^{2}}^{2}$ & $\begin{array}{l}6 / \mathrm{ml}^{2} \mathrm{~F} 6 / \mathrm{m} \\
6 / \mathrm{m} \mathbb{1}^{2} \mathrm{~F} 6^{2} / \mathrm{m}^{2}\end{array}$ & $\ldots$ \\
\hline & $\frac{E_{2 z^{2}}}{E_{2 z^{2}}}$ & $\begin{array}{l}6 / \mathrm{m} 1^{2} \mathrm{~F} 2^{2} / \mathrm{m}^{2} \\
6 / \mathrm{m} 1^{2} \mathrm{~F} 2 / \mathrm{m}\end{array}$ & $\ldots$ \\
\hline & $A_{2}^{2}$ & $6 / \mathrm{mll}^{2} \mathrm{~F} 6 / \mathrm{m}^{2}$ & $\left(\frac{x x^{2}+x y^{2}}{2}, x y^{2}, z z^{2}\right) 1$ \\
\hline & $\mathrm{B}_{2}^{2}$ & $6 / \mathrm{ml}^{2} \mathrm{~F} 6^{2} / \mathrm{m}$ & $\ldots$ \\
\hline & $\mathrm{E}_{\mathrm{iv} \mathrm{u}^{2}}$ & $6 / \mathrm{m} 1^{2} \mathrm{~F} 2^{2} / \mathrm{m}$ & $\left(x z^{2}, y z^{2}, z x^{2}, z y^{2}\right) 1$ \\
\hline & $\mathrm{E}_{2 u^{2}}{ }^{2}$ & $6 / \mathrm{ml}^{2} \mathrm{~F} 2 / \mathrm{m}^{2}$ & $\begin{array}{l}\left(x x^{2}, y x^{2}\right) 1 \\
\left(x y^{2}, x y^{2}, z z^{2}\right) \quad 3\end{array}$ \\
\hline \multirow[t]{5}{*}{$6221^{1}$} & $\mathbf{A}_{2}{ }^{2}$ & $62211^{2} \mathrm{~F} 622$ & $\left(\frac{x x^{2}+x y^{2}}{2}, z z^{2}\right) \quad 1$ \\
\hline & $A_{2}{ }^{2}$ & $6221^{2} \mathrm{~F} 62^{2} 2^{2}$ & $\left(x v^{2}\right)$ where $\left(\lambda_{21}=-\lambda_{12}\right) \quad 1$ \\
\hline & $\mathrm{B}_{\mathrm{s}}^{2}(\mathrm{i}=1,2)$ & $6221^{2} \mathrm{~F}^{2} 22^{2}$ & $\ldots$ \\
\hline & $E_{2}{ }^{2}$ & $6221^{2} \mathrm{~F} 22^{2}$ & $\left(x z^{2}, y z^{2}, z x^{2}, z y^{2}\right) 1$ \\
\hline & $\mathrm{E}_{2}^{2}$ & $6221^{2} \mathrm{~F} 2$ & $\begin{array}{l}\left(x x^{2}, x y^{2}\right) 1 \\
\left(x x^{2}, x y^{2}, z z^{2}\right) 3\end{array}$ \\
\hline \multirow[t]{5}{*}{$6 \mathrm{mmil}^{1}$} & $\mathbf{A}_{2}{ }^{2}$ & $6 \mathrm{~mm} 11^{2} \mathrm{~F} 6 \mathrm{~mm}$ & $\left(x y^{2}\right)$ where $\left(\lambda_{z 1}=-\lambda_{1 x}\right) 1$ \\
\hline & $A_{2}{ }^{2}$ & $6 \mathrm{~mm} 1^{2} \mathrm{~F} 6 \mathrm{~m}^{2} \mathrm{~m}^{2}$ & $\left(\frac{x x^{2}+x y^{2}}{2}, z z^{2}\right) \quad 1$ \\
\hline & $\mathrm{B}_{\mathrm{s}}^{2}(\mathrm{i}=1,2)$ & $6 m m 1^{2} F 6^{2} m^{2} m$ & $\ldots$ \\
\hline & $\mathrm{E}_{\mathrm{t}}{ }^{2}$ & $6 \mathrm{~mm} 1{ }^{2} \mathrm{~F} 2^{2}$ & $\left(x z^{2}, y z^{2}, z x^{2}, z y^{1}\right) 1$ \\
\hline & $\mathrm{E}_{2}{ }^{2}$ & $6 m m 1{ }^{2} F 2$ & $\begin{array}{l}\left(x x^{2}, x y^{2}\right) 1 \\
\left(x x^{2}, x y^{2}, z z^{2}\right) 3\end{array}$ \\
\hline \multirow[t]{6}{*}{$62 \mathrm{mal}^{1}$} & $A_{\text {ix }^{2}}$ & $62 \mathrm{ml}^{1} \mathrm{~F} 62 \mathrm{~m}$ & $\ldots$ \\
\hline & $A_{2 x^{2}}{ }^{2}$ & $62 \mathrm{ml}^{1} \mathrm{~F} 62^{1} \mathrm{~m}^{2}$ & $\ldots$ \\
\hline & $E_{2}{ }^{2}$ & $62 \mathrm{mll}^{1} \mathrm{Fm}$ & $\left(x z^{2}, y z^{2}, z x^{2}, z y^{2}\right) 1$ \\
\hline & $A_{1: 2}{ }^{2}$ & $62 \mathrm{ml}^{1} \mathrm{~F} \overline{6}^{2} 2 \mathrm{~m}^{2}$ & $\left(\frac{x x^{2}+y y^{2}}{2}, x z^{2}\right) \quad 1$ \\
\hline & $A_{2 x^{2}}$ & $\overline{6} 2 \mathrm{ml}^{1} \mathrm{~F}^{2} 2^{1} \mathrm{~m}$ & $\left(x v^{2}\right)$ where $\left(\lambda_{21}=-\lambda_{2 x}\right) 1$ \\
\hline & $\mathrm{E}_{2}{ }^{22}$ & $62 \mathrm{mll}^{1} \mathrm{Fm} \mathrm{m}^{2}$ & $\begin{array}{l}\left(x x^{2}, x y^{2}\right) 1 \\
\left(x x^{2}, x v^{2}, z z^{2}\right) 3\end{array}$ \\
\hline \multirow[t]{4}{*}{$6 / \mathrm{mmmmi}^{1}$} & $A_{1 z^{2}}$ & 6/mmm1 ${ }^{2}$ F 6/mm & $\cdots$ \\
\hline & $A_{2 z^{2}}$ & 6/mmm I ${ }^{2}$ F $6 / \mathrm{mmm}^{2} \mathrm{~m}^{2}$ & $\ldots$ \\
\hline & $\begin{array}{l}\mathrm{B}_{z_{z}}{ }^{2}(i=1,2) \\
\mathrm{E}_{u^{2}}\end{array}$ & $6 / \mathrm{mmm} 1^{2} \mathrm{~F} 6^{2} / \mathrm{m}^{2} \mathrm{~m}^{2} \mathrm{~m}$ & $\cdots$ \\
\hline & $E_{2 z} z_{z}^{2}$ & $6 / \mathrm{mmm1}^{2} \mathrm{~F} 2 / \mathrm{m}$ & $\ldots$ \\
\hline
\end{tabular}


Tensor Components and indices of faintness for magneto-electric ferroic species

\begin{tabular}{|c|c|c|c|}
\hline & $A_{2=x^{2}}$ & $6 / m m m 11^{2} \mathrm{~F} 6 / \mathrm{m}^{2} \mathrm{~m}^{2} \mathrm{~m}^{2}$ & $\left(\frac{x x^{2}+y y^{2}}{2}, z z^{2}\right) \quad 1$ \\
\hline & $A_{2 x^{2}}$ & $6 / \mathrm{mmml}^{2} \mathrm{~F} 6 / \mathrm{m}^{2} \mathrm{~mm}$ & $\left(x v^{2}\right)$ where $\left(\lambda_{21}=-\lambda_{12}\right) 1$ \\
\hline & $\mathrm{B}_{\mathrm{sin}}{ }^{2}(i=1,2)$ & $6 / \mathrm{mmm}^{2} \mathrm{~F}^{2} / \mathrm{mm}^{2} \mathrm{~m}$ & $\ldots$ \\
\hline & $E_{1 u^{2}}$ & $6 / \mathrm{mmm} 1^{2} \mathrm{~F} 2^{2} / \mathrm{m}$ & $\left(x z^{2}, y z^{2}, z x^{2}, z y^{2}\right)$ \\
\hline & $\mathrm{E}_{2 \mathrm{ut}}{ }^{2}$ & $6 / \mathrm{mmm}^{2}{ }^{2} \mathrm{~F} 2 / \mathrm{m}^{2}$ & $\begin{array}{l}\left(x x^{2}, w x^{2}\right) 1 \\
\left(x y^{2}, y y^{2}, z z^{2}\right) 3\end{array}$ \\
\hline \multirow[t]{3}{*}{$231^{1}$} & $A^{2}$ & $231^{2} \mathrm{~F} 23$ & $\left(\frac{x x^{2}+y y^{2}+z z^{2}}{3}\right) 1$ \\
\hline & $\mathbf{E}^{2}$ & $231^{2} \mathrm{~F} 2$ & $\begin{array}{l}\left(x y^{2}, w x^{2}\right) 1 \\
\left(x x^{2}, w w^{2}, z z^{2}\right) \mathbf{B}\end{array}$ \\
\hline & $\mathrm{T}^{2}$ & $231^{2} \mathrm{~F} 1$ & $\begin{array}{l}\left(x z^{2}, w y^{2}, w z^{2}, z x^{2}, x y^{2}, z z^{2}\right) 1 \\
\left(x x^{2}, x y^{2}, v x^{2}\right) 3^{3}\end{array}$ \\
\hline \multirow[t]{6}{*}{$\operatorname{m3} 1^{1}$} & $A_{z}{ }^{2}$ & $\mathrm{~m} 31^{2} \mathrm{Fm} 3$ & $\ldots$ \\
\hline & $\mathrm{E}_{z}{ }^{2}$ & $\mathrm{~m} 31^{2} \mathrm{~F} 2 / \mathrm{m}$ & $\ldots$ \\
\hline & $\mathrm{T}_{z}{ }^{2}$ & $m 31^{2} \mathrm{Fi}$ & $\ldots$ \\
\hline & $A_{x}{ }^{2}$ & $\mathrm{~m} 31^{2} \mathrm{Fm}^{2} 3$ & $\left(\frac{x x^{2}+y y^{2}+z z^{2}}{3}\right) \mathbf{1}$ \\
\hline & $E_{x^{2}}{ }^{2}$ & $\mathrm{~m} 31^{2} \mathrm{~F} 2 / \mathrm{m}^{2}$ & $\begin{array}{l}\left(x y^{2}, w x^{2}\right) 1 \\
\left(x x^{2}, w w^{2}, z z^{2}\right)^{3}\end{array}$ \\
\hline & $T_{x^{2}}=$ & $m 31^{2} \mathrm{Fi}^{2}$ & $\begin{array}{l}\left(x x^{2}, x z^{2}, y y^{2}, y z^{2}, 2 x^{2}, z y^{2}\right) 1 \\
\left(x y^{2}, y x^{2}, z z^{2}\right) 3\end{array}$ \\
\hline \multirow[t]{4}{*}{$4321^{1}$} & $A_{2}{ }^{2}$ & $4321^{2} \mathrm{~F} 432$ & $\left(\frac{x x^{2}+x y^{2}+x z^{2}}{3}\right), 1$ \\
\hline & $A_{2}{ }^{2}$ & $4321^{2} \mathrm{~F}^{2} 32^{2}$ & $\ldots$ \\
\hline & $E^{2}$ & $4321^{2} \mathrm{~F} 2$ & $\begin{array}{l}\left(x v^{2}, w x^{2}\right) 1 \\
\left(x x^{2}, w w^{2}, x z^{2}\right) \mathbf{B}\end{array}$ \\
\hline & $T_{i}^{2}(i=1,2)$ & $4321^{2} \mathrm{~F} 1$ & $\begin{array}{l}\left(x x^{2}, x z^{2}, w y^{2}, y z^{2}, x x^{2}, z y^{2}\right)^{1} \\
\left(x y^{2}, w x^{2}, z z^{2}\right)^{3}\end{array}$ \\
\hline $43 \operatorname{mn} \mathrm{T}$ & $A_{2}{ }^{2}$ & $43 \mathrm{mll}^{1} \mathrm{~F}^{4} 3 \mathrm{~mm}$ & $\ldots$ \\
\hline
\end{tabular}

\begin{tabular}{|c|c|c|c|}
\hline & $\mathrm{A}_{2}{ }^{1}$ & $\overline{4} 3 \mathrm{ml}^{1} \mathrm{~F}^{-1} 3 \mathrm{~m}^{1}$ & $\left(\frac{x x^{1}+y y^{1}+z z^{1}}{3}\right) \mathbf{1}$ \\
\hline & $\mathrm{E}^{1}$ & $\overline{4} 3 \mathrm{ml}^{1} \mathrm{~F} 2$ & $\begin{array}{l}\left(x y^{1}, y x^{1}\right) \mathbf{1} \\
\left(x x^{1}, y y^{1}, z z^{1}\right)^{3}\end{array}$ \\
\hline & $\mathrm{T}_{\mathrm{i}}^{1}(\mathrm{i}=1,2)$ & $\overline{4} 3 \mathrm{~m}^{1} \mathrm{~F} 1$ & $\begin{array}{l}\left(x z^{1}, y y^{1}, y z^{1}, z x^{1}, z y^{1}, z z^{1}\right) \mathbf{1} \\
\left(x x^{1}, x y^{1}, y x^{1}\right) \mathbf{3}\end{array}$ \\
\hline \multirow[t]{8}{*}{$\mathbf{m} 3 \mathbf{m l}^{1}$} & $\mathrm{~A}_{1 \mathrm{~g}^{1}}$ & $\mathrm{~m} 3 \mathrm{~m} 1^{1} \mathrm{Fm} 3 \mathrm{~m}$ & $-\cdots$ \\
\hline & $\mathrm{A}_{2 \mathrm{~g}^{1}}$ & $\mathrm{~m} 3 \mathrm{~m} 1^{1} \mathrm{Fm} 3 \mathrm{~m}^{1}$ & $-\cdots$ \\
\hline & $\mathrm{E}_{\mathrm{g}} \mathrm{I}^{\mathrm{l}}$ & $\mathrm{m} 3 \mathrm{~m}^{1} 1^{1} \mathrm{~F} 2 / \mathrm{m}$ & $-\cdots$ \\
\hline & $\mathrm{T}_{\mathrm{ig}}{ }^{1}(\mathrm{i}=1,2)$ & $\mathrm{m} 3 \mathrm{~m} 1^{1} \mathrm{~F} \overline{1}$ & $\ldots$ \\
\hline & $\mathrm{A}_{1 \mathrm{u}^{1}}$ & $\mathrm{~m} 3 \mathrm{~m}^{1}{ }^{1} \mathrm{Fm}^{1} 3 \mathrm{~m}^{1}$ & $\left(\frac{x x^{1}+y y^{1}+z z^{1}}{3}\right) \mathbf{1}$ \\
\hline & $\mathbf{A}_{2 u^{1}}$ & $\mathrm{~m} 3 \mathrm{~m}^{1} \mathrm{Fm}^{1} 3 \mathrm{~m}$ & $-\cdots$ \\
\hline & $E_{u}^{1}$ & $\mathrm{~m} 3 \mathrm{~m} 1^{1} \mathrm{~F} 2 / \mathrm{m}^{1}$ & $\begin{array}{l}\left(x y^{1}, y x^{1}\right) \mathbf{1} \\
\left(x x^{1}, y y^{1}, z z^{1}\right) \mathbf{3}\end{array}$ \\
\hline & $\mathrm{T}_{\mathrm{iu}}{ }^{1}(\mathrm{i}=1,2)$ & $\mathrm{m} 3 \mathrm{~m} 1^{1} \mathrm{~F} \overline{1}^{-2}$ & $\begin{array}{l}\left(x x^{1}, x z^{1}, y z^{1}, z x^{1}, z y^{1}, z z^{1}\right) \mathbf{1} \\
\left(x y^{1}, y x^{1}, y y^{1}\right)^{\mathbf{3}}\end{array}$ \\
\hline
\end{tabular}

II. Conclusions:

The faintness indices of a degenerate set of soft vibrational modes for ferroelectricity and ferroelasticity are evaluated by Aizu. In this present paper magneto-electric polarizability property is considered. The ferroic 
species are associated to their corresponding irreducible representations for all 32 grey groups. The faintness indices for the magneto-electric polarizability components of corresponding ferroic species are obtained.

\section{References:}

[1] S. Bhagavantham, (1966). "Crystal Symmetry and physical properties". Academic press, London, p. 167 - 172

[2] Bradley, C.J and Cracknell, (1972). "Mathematical theory of symmetry in solids”. Clarendon press, Oxford, p. 570 - 572

[3] W.A. Wooster, (1973). "Tensors and group theory for the physical properties of crystals". Clarendon press, Oxford, p. 113 - 118

[4] Keitsiro Aizu.(1973). “Journal of the physical society of Japan”. Vol. 35 p : 181-187 and 1704 - 1707

[5] K. Aizu. (1972). "Journal of the physical society of Japan". vol. 33 p : 1390 